\title{
Office Examination of Palatopharyngeal Function
}

\author{
H. HARLAN BLOOMER, PH.D., * WILLIAM WOLSKI, PH.D.**
}

AHILDREN are continually being referred to speech clinics or public school speech correction programs because of previously unrecognized or undiagnosed defects of the palatopharyngeal valve. Some of these defects are of congenital origin, others result from disease or have followed surgical removal of the tonsils and adenoids. If allowed to go untreated, these children may be handicapped for life; whereas early detection of actual or potential palatal inadequacy or insufficiency can lead to successful treatment during the important developmental years.

\section{Palatopharyngeal Closure}

The soft palate and its associated tissues of the nasopharynx and oropharynx participate significantly in breathing, speaking, hearing, sucking, swallowing, whistling and blowing. Every one of these is important to the integrity and normal life-adjustment of the individual, yet the symptom for which a patient most often seeks advice is palatal inadequacy affecting speech. One must emphasize here that the mistaken diagnosis of palatopharyngeal function may lead to misdirected medical treatment or surgery, and especially the unnecessary removal of tonsils and adenoids. On the other hand, if the examining physician fails to recognize the full import of the symptoms of palatal abnormalify the start of appropriate corrective proce-

\footnotetext{
* Professor of Speech, College of Literature, Science and the Arts, and Professor of Otorhinolaryngology, Medical School, The University of Michigan, 1111 East Catherine Street, Ann Arbor, Michigan 48104.

* Chief, Department of Speech and Tearing, G. S. Mott Foundation Children's Health Center, 806 West Sixth Avenue, Flint, Michigan 48503.
}

A functional palatopharyngeal valve is es. sential for the production of normal speech. Any malfunctioning of this valve, as indicated by hypernasality, hyponasality, or impaired speech intelligibility, warrants careful examination and diagnosis.

For a complete study of the palate and its associated tissues one needs a specialist, but simple procedures for identification of palatopharyngeal dysfunction can be carried out by the physician in general practice. These procedures include evaluation of speech, visual examination of the palate and nasopharynx, digital palpation of the hard and soft palate, soft tissue $x$-ray films of the head, and the use of readily obtainable instruments such as a steel mirror, stethoscope, and spirometer. Patients who have inadequate palatopharyngeal function and abnormal speech should be referred for special examination and treatment.

This paper identifies some of the causes of palatopharyngeal dysfunction, describes the procedures which can be used in its detection, and suggests referral sources available for special consultation.

dures will be delayed and the development of normal speech may be seriously retarded.

Voluntary control of the palate and its associated structures contributes to normal speech in two ways: (1) it enables the speaker to utilize nasal resonance selectively, and (2) it enables him to build up sufficient oral air pressure to articulate clearly the nonnasal sounds of speech. A defective palatopharyn. geal valve may lead to speech which is hypernasal or hyponasal or subject to articulatory distortion, each of which will affect the intelligibility and social acceptability of speech. Such deficiency in control may also hamper 


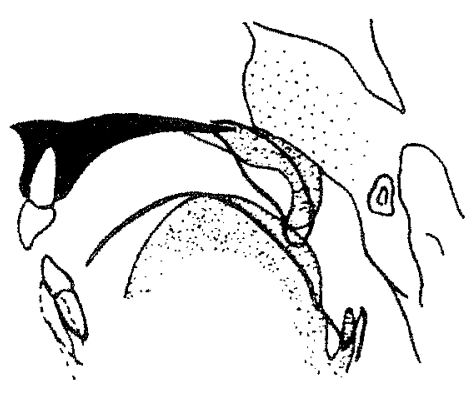

DENASAL SPEECH

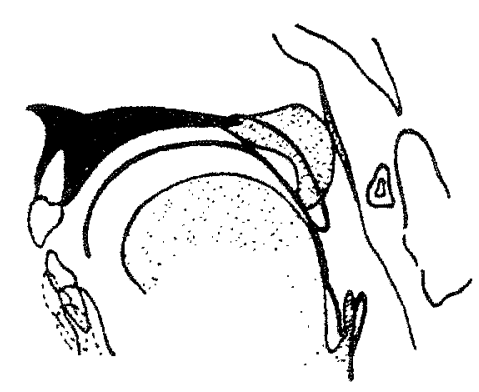

NORMAL SPEECH
FIG. 1. Pre- and postoperative tracings of cephalometric lam. inographs of the same individual under two conditions: at rest (solid black lines) and dur. ing plonation (stippled areas of palate and tongue). Note that pre-operative position of the adenoid pad nearly occludes the nasopharyngeal space. Such a condition discourages nasal breathing and creates "adenoidal" (hyponasal) speech. (Ref. Nio. 3, p. 244.) the other physiologic activities mentioned above.

The only sounds of spoken English which are normally produced with nasal resonance are $/ \mathrm{m} /, / \mathrm{n} /$, and $/ \mathrm{ng} /$. Only during the production of these sounds does the palatopharyngeal port open in order to permit the nasopharynx and the nasal passages to act as significant resonating chambers. For all of the other approximately 22 consonants and 14 vowels and diphthongs, the palatopharyngeal port remains closed and the airflow and the resonances of speech are directed via the oropharyngeal and oral route.

Failure of such palatopharyngeal closure results in distortion of most speech sounds, oftentimes to serious interference with intel. ligibility. The articulation of plosive consonants such as $/ \mathrm{p} /, / \mathrm{b} /, / \mathrm{t} / \mathrm{d} / \mathrm{h} / \mathrm{k} /$, or /g/ will be imperfect. The speaker may be making the requisite lip and tongue contacts for correct articulation, but the leakage of a significant part of the breath stream through the nasal passages will not only weaken the oral pressures needed for intelligible utterance but will add nasal resonance, thus causing the labial and lingual sounds to resemble $/ \mathrm{rn} / \mathrm{s} / \mathrm{n} /$, or $/ \mathrm{ng} /$. "Bad" may resemble "man," "dot" may sound somewhat like "non," and so forth. Similar distortions will affect the intelligibility of other sounds, including even the consonant glides $/ 1 /, / r /$ or $/ y /$.

Palatopharyngeal inadequacy does not accoumt for all of the deviant articulation patterns in obvious hypernasality with defective palatal function. A patient's use of aberrant lingual positioning or movements may be a major cause. Such cases will not be improved immediately by the creation of a functional palatopharyngeal valve, though this may assist indirectly. One effective test with such a person is to occlude his nostrils during his enunciation of test words. If this significantly improves intelligibility, nasal leakage may be identified as the major contributant.

Whereas hypernasality is almost always attributable to palatopharyngeal valve dysfunction or deficiency, hyponasality (absence of nasal resonance) may be due to any of several factors. These include inappropriate and involuntary contraction of the valve (as occurs at times in cerebral palsy); enlarged adenoids; constriction or stenosis of the nasal passages or the nasopharyngeal space by tumor, polyp, edema; constriction or contraction of the anterior nares.

\section{Dynamics of Palatopharyngeal Function}

Control of the palatopharyngeal port entails interaction of several muscle pairs-the tensor veli palatini, levator veli palatini, constrictor pharyngeus superior, salpingopharyngeus, palatopharyngeus, palatoglossus and possibly stylopharyngeus. The tensor muscle acts particularly during swallowing, cooperating with the tongue in squeezing the bolus backward toward the pharynx, and it may on occasion contract in connection with the speech acts. The uvular muscles, al though active during speech, probably play little part in palatopharyngeal valving, and play little role in 


\section{PALATOPHARYNGEAL FUNCTION}

A NON-CLEFT PALATE PATIENT

Frg. 2. Nasal speech due to adenoidectomy in a patient with palatal insufficiency. Although the soft palate elevates and retracts after adenoidectomy it is unable to close the valve postoperatively. The shaded area indicates the approximate position of adenoid tissue prior to removal. (Modified from Ref. No. 3, p. 249.)
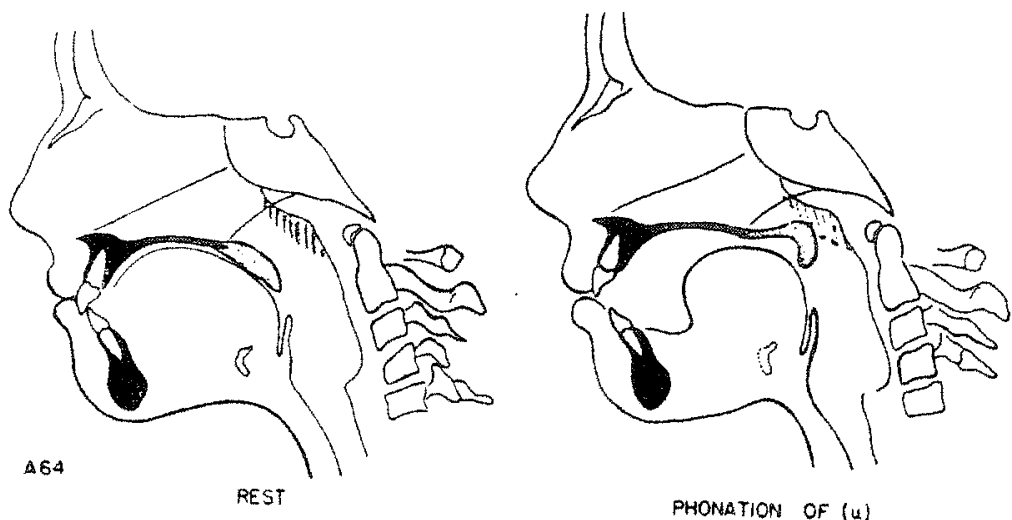

speech except in the production of a uvular $/ \mathrm{r} /$.

The cranial motor innervation of these muscles is through branches of the vagus, except for the stylopharyngeus and the tensor veli palatini which are supplied, respectively, by the glossopharyngeal and trigeminal nerves. Action of these muscles has been well illustrated by Podvinec. ${ }^{2}$

\section{Causes and Types of Palatopharyngeal Dysfunction}

Failure to achieve adequate closure for speech may occur for two basic reasons: (1) inadequate tissue substance (designated as palatal insufficiency); or (2) inadequate movement (palatal incompetence). A typical instance of insufficiency is that due to cleft or submucous cleft of the palate; of incompetency, paralysis of palatal musculature.

The causes of palatal insufficiency or incompetence are various, acting singly or in combination. Foremost among the causes are: (1) open or submucous palatal clefts; (2) palatal tissue deficiency (short palate of congenital or postnatal origin); (3) muscle weakness due to genetic factors or paralysis following bulbar poliomyelitis, myasthenia gravis, amyo trophic lateral sclerosis or other damage to the central or peripheral nervous system; (4) abnormal depth of the pharyngeal space (sometimes congenital, or surgicaliy created by adenoidectomy); (5) scar tissue restricting the range of palatal movement following sur. gery or injury; (6) dysfunction associated with congenital heart defect or other debilitating disease; and (7) habits related to imitation of the hypernasal speech of others, or to the persistence of muscle movement patterns established prior to palatopharyngeal surgery.

Some of these conditions have a poor treatment prognosis. Others are avoidable. The dysfunction which sometimes follows adenoidectomy can often be avoided if proper steps are taken in pre-operative examination and postoperative care. In children whose palate is short or inactive, the adenoid pad aids in narrowing the nasopharyngeal space. This is well illustrated in the studies of Subtelny and Koepp-Baker, ${ }^{3}$ who traced by roentgenography the course of adenoid development from infancy to young adulthood. With such instances, the adenoid pads should not be removed unless they are diseased suffciently to affect the total health of the child.

Occasionally a child is brought to the speech pathologist with the report that an adenoidectomy was done because a physician thought "it might help their speech." With some such children the speech defect is actually increased by the operation and the task of the speech pathologist made more diffcult because, as shown in Figure 2, the child can no longer achieve palatopharyngeal closure. Hyponasal voice equality or defective speech articulation are never by themselves indicators of need for adenoid removal. However, diseased adenoids and tonsils which interiere with hearing or the general health of the child should of course be treated, regardless of the possible implications for valve function.

Even in normal subjects, surgical removal of tonsils and adenoids may produce unfor- 


\section{BLOOMER AND WOLSKI}

tunate and permanent results. In these instances, the postoperative palate on oral inspection may appear normal. More detailed studies may show, however, a partial paralysis (sometimes hemilateral) or scarring which partially inhibits palatal elevation and retraction during speech. Deep dissection of posterior nasopharyngeal tissues may have created a nasopharynx too deep to be closed by normal palatal movement. A child may retain the preoperative pattern of minimal palatal movement, and fail after surgery to make the extra contraction now required to close the palatopharyngeal valve.

Palatopharyngeal incompetence in the $a b$ sence of organic causes can usually be corrected by speech therapy, including appropriate exercises to establish voluntary control of palatopharyngeal movement. When tissue deficiency exists or innervation has been impaired, surgical or prosthetic correction will be required.

\section{Examination Procedures}

The examination of palatopharyngeal function should be a part of routine physical appraisal, and especially when the child has any speech, voice, or feeding difficulty. Evaluation of the palatopharyngeal mechanism may not be easy, however: (1) ordinary oral inspection does not permit the palatopharyngeal port to be seen during function, (2) the performance is often inconsistent, (3) special technics are required to measure or infer the degree and character of function of the palatopharyngeal valve.

The following sections describe a number of useful procedures which can be used by the physician in his office. These include: (1) auditory evaluation of the patient's voice quality and articulation during conversational speech and selected speech samples; (2) visual examination by indirect nasopharyngoscopy; (3) digital palpation of the hard and soft palate, and (4) whe use of instruments to register airlow or air volume, such as: (a) a spirometer, (b) a steel mirror (rhino-hygrometer), and (c) a modified stethoscope. These procedures, singly, or in combination, are easy to do and require equipment which should be available in every physician's office. Any abnormalities found in these examinations can lead to selective referral for additional evaluative procedures, such as $\mathrm{x}$-ray studies.

\section{Auditory Evaluation}

Preliminary judgments as to adequacy of palatopharyngeal action may be made by listening to a patient's speech. As noted, hypernasal voice quality almost always indicates inadequate closure, whereas hyponasality may result either from hypercontraction of the palatopharyngeal port or from blockage of the nasal passages. Acoustic cues may be subtle and difficult to quantify, but usually provide the first alerting signs of palatopharyngeal dysfunction.

Observations of hypernasality in spontaneous speech responses may be verified by asking the patient to repeat words and sentences which have no nasal consonants. For example: "Buy baby a bib," "Zippers are easy to close," "Go get a bigger egg," count "two, three, four, five, six," or say "ee-oh-ee-oh." If the voice quality for these speech samples is changed when the patient repeats them with his nostrils manually occluded, he is judged to be a hypernasal speaker. (Pinching the nose does not affect normal voice production of "nonnasalized" sounds.) When the palatopharyngeal valve does not close, the quality change caused by manual nasal occlusion becomes apparent immediately.

Sentences or words rich in nasal sounds may be used to test for hyponasality. For this test the patient is asked to say, "Mama made some lemon jam," or to count from "ninety to one hundred" by ones. Hyponasal voice sounds "stuffy" and "adenoidal," and quality may be little affected by manual occlusion of the nostrils.

\section{Visตล Examination}

This should start with direct looking for external (facial) signs of palatopharyngeal falure, nsully in the form of facial grimaces. In particular, a "pinching in" of the nares during speech often occurs as the patient attempes valving at the nares to compensate for lack of closure at the palatopharyngeal port. It can frequently be observed in the "cleft palate" speech of patients whose surgi- 


\section{PALATOPHARYNGEAL FUNCTION}

cal treatment has failed to produce a functional palatopharyngeal valve.

Closure of the palatopharyngeal valve cannot be observed directly by oral inspection. But having the patient open his mouth to phonate "ah" while the tongue is depressed enables the examiner to observe the degree and character of movement of the palatopharyngeal tissues. The normal palate should elevate and retract noticeably above the position of drape usual during relaxation.

Extent of movement and bilateral symmetry of movement should be noted in elevation and retraction of the palate and in medial approximation of lateral pharyngeal tissues. Even a small opening of the valve during phonation may cause objectionable hypernasality. It is of special importance for the examiner to note whether the patient is able to sustain the velum in elevation as long as phonation is continued. Some patients make a good initial response, but the valve relaxes perceptibly as phonation is sustained for several seconds, with a resultant intrusion of hypernasality. This relaxation of the valve is often a sign of muscle weakness, as is progressive increase in hypernasality throughout the day.

Whereas the actual site of closure ordinarily occurs well above the line of vision, a nasopharyngeal mirror may be used to observe the relative positions of the soft tissues of the nasopharynx during attempted palatal contraction. If closure is incomplete, the degree of patency may be seen in the mirrored image, especially in cases of palatal cleft, tissue deficiency or partial paralysis.

Another test is to shine a guarded light source into an. open nostril with the patient in a darkened room. Abnormally thin palatal tissues will be translucent in comparison to tissues of normal thickness and musculature, and light reffected onto the oropharyngeal wall will indicate failure of palatopharyngeal closure.

\section{Palpation}

One should palpate the hard and soft palate to check for submucous clefts, running the finger along the roof of the mouth to the posterior border (nasal spine) of the hard palate. Translucency of the soft palate may indicate also a submucous cleft or the absence of adequate muscle tissues.

Elevation of the soft palate by a tongue blade as the patient sustains phonation of "ah" provides an effective way to determine whether a change in voice quality occurs as a result of change in position of the palate. If the voice quality is noticeably changed by palatal elevation, this provides further indication that the palatopharyngeal function is defective.

\section{Rhino-Hygrometer}

A simple but effective means of detecting nasal emission of air employs a metal mirror as a rhino-hygrometer. The mirror should be cold, or at least cooler than the ambient air. A common Boy Scout steel mirror is adequate for this test.

One first notes normal patterns of emission by placing the mirror beneath the child's nose as he breathes quietly and as he hums. The nasal air normally emitted during these activities forms condensation patterns on the upper surface of the mirror. The amount of condensation provides a reference basis for comparison with the clegree of nasal emission which may occur as a patient attempts to produce speech sounds which are normally nonnasalized. Conversely, a lack of moisture condensation during humming or the speaking of words containing nasal sounds (e.g., "mama," "many," "New pennies shine") will indicate obstruction in the nasopharynx or nasal passages.

Once a moisture condensation pattern characteristic of maximum nasal patency has been established, the patient should be asked to sustain speech sounds such as "ah," "ee," "ssss," and "ffff" for several seconds. The mirror is placed beneath the nostrils during the production of these sounds or during the enunciation of sentences lacking nasal consonants. Comparisons between condensation patterns for these sounds and those obtained for humming permits a roughly quantitative judgment as to the degree of nasal air emission. If a nasal passage is occluded there will be, of course, no condensation on the obstructed side. 


\section{BLOOMER AND WOLSKI}

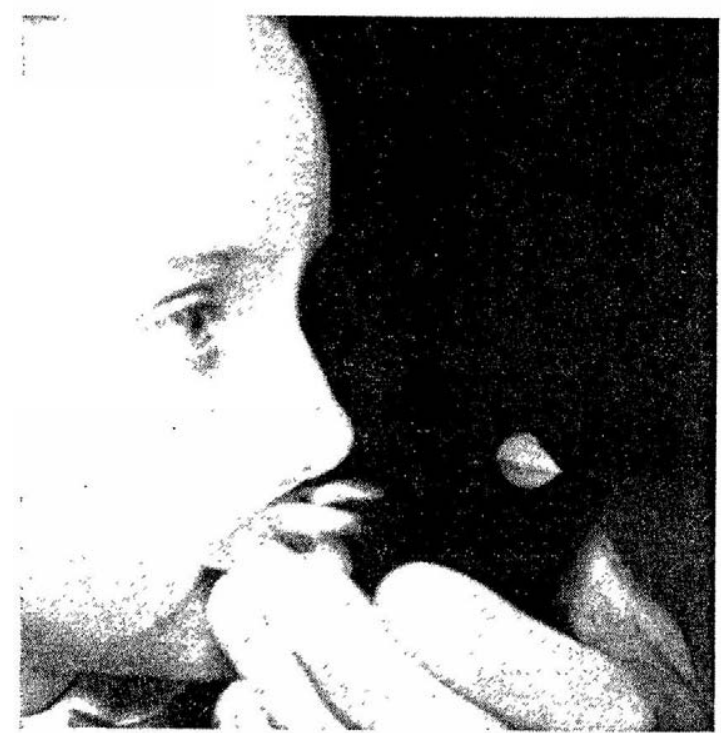

Fic. 3. Normal patterns of nasal emission during humming are recorded as white "patches" of condensation on the upper surface of the mirror, directly under the nostrils.

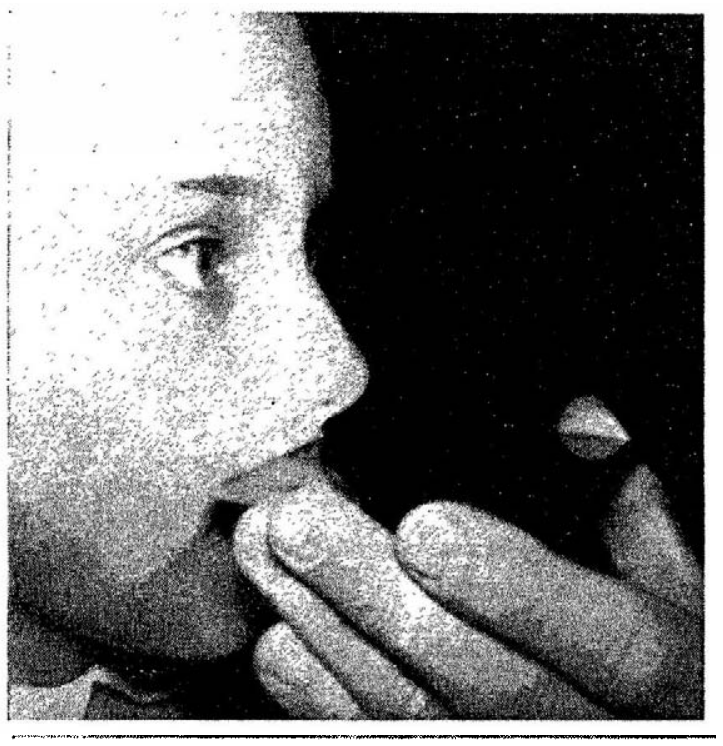

Fic. 4. Normal palatopharyngeal function for suscained /ab/ sound is evidenced by the absence of condensation patterns on the upper surface of the mirror.

The examiner should be careful to place the mirror in position afte? phonation has been initiated, and to withdraw it before phonation has stopped. This eliminates the possibility of recording the nasal exhalation which normally occurs in breathing and at the completion of an utterance.

The ability to sustain closure should also be tested. Some patients may initially elevate

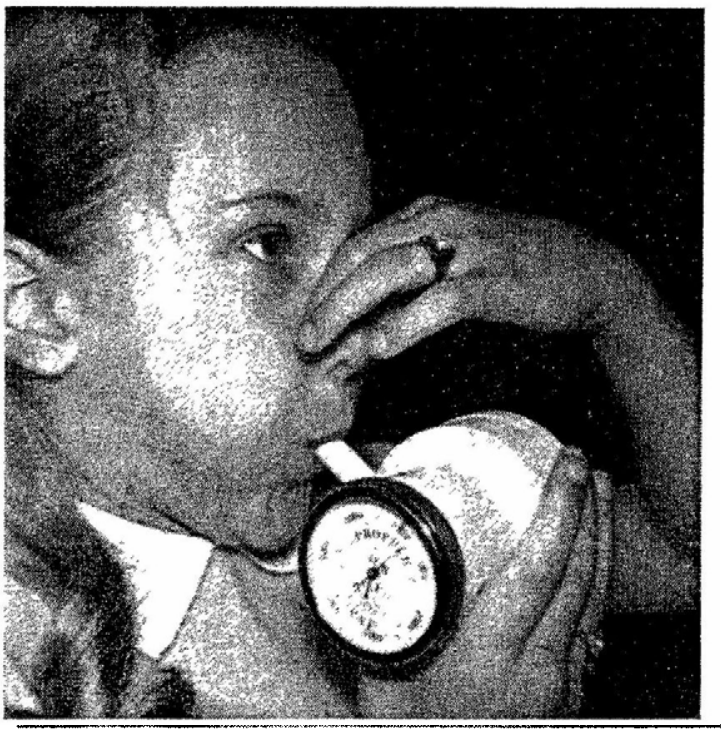

Fic. 5. The patient holds her nose while blowing to produce an aided score on the volume scale of the dry spirometer.

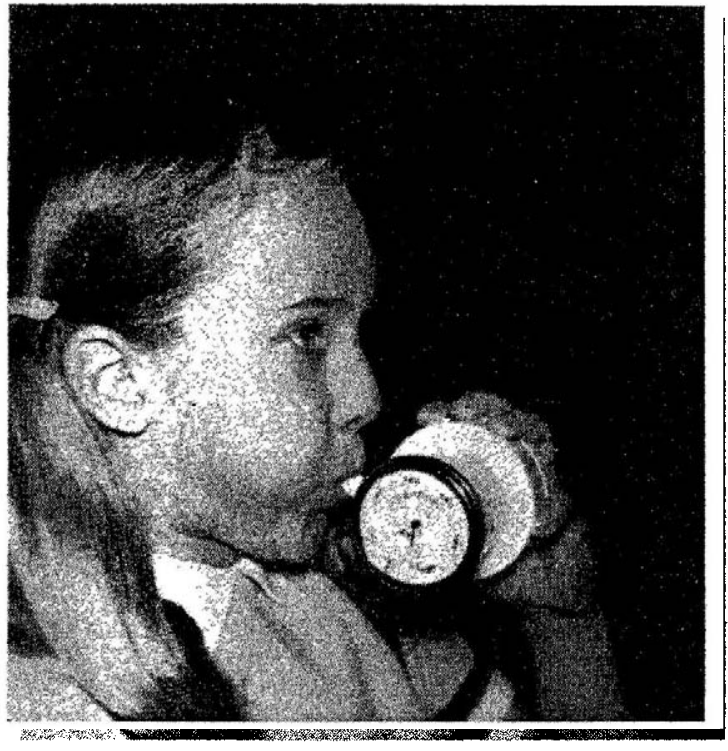

Fic. 6. The blowing procedure is repeated with the nostrils open, producing an undided score.

and retract the palate (as noted under "Visnal Examinaton" above), but thereafter relax it quickly, thereby opening the palatopharyngeal valve. This occurs especially with weakness or congenital defects of the palatopharyngeal muscles.

It also sometimes happens that a child succeeds in closing the valve for isolated speech sounds but fails to do so for the more de- 


\section{PALATOPHARYNGEAL FUNCTION}

manding, dynamic process of uttering words and sentences. For this reason, it is important to test palatopharyngeal closure during the speaking of sentences which contain no nasal sounds, as suggested earlier in the section on "Auditory Evaluation."

\section{Stethoscopic Test}

The stethoscopic test is a very sensitive one, and provides a means of refining and validating the ordinary tests of listening.

The bell of a stethoscope is replaced by a glass tube, nasal olive or even a short section of a drinking straw of appropriate size. The examiner places the tube or straw at the orifice of the patient's nostrils as he speaks or produces sustained speech sounds. Nasal emission and nasal resonance, will be heard as air passes into or across the opening of the tube. The same kinds of testing activities may be used as described for the hygrometer tests. Judgments of the presence or absence of nasal air emission and nasal resonance may thus be made.

\section{Spirometer}

The activities of the palatopharyngeal valve in speaking and in blowing are similar, ${ }^{1}$ although blowing usually stimulates a greater contraction of the palatopharyngeal muscles than occurs during speech.

A spirometer used in estimating physical fitness and pulmonary and cardiac efficiency provides a means of estimating palatal effciency for blowing. An index of palatal efficiency is obtained by comparing the volume achieved for blowing with the nostrils occluded (aided score) with that obtained with the nostrils open (unaided score). A wet or dry spirometer may be used. The latter, however, of either a bellows or meter type is more compact, portable, and convenient.

To test palatal efficiency for blowing, first instruct the patient to "Take as big a breath as possible, hold your nose, and blow all the air you can through the mouthpiece." This procedure is repeated two or three times, and the highest reading on the spirometer's vol. ume scale is recorded as the aided score. The patient is asked to repeat this procedure $u n$ aided (with his nostrils open). The percentage of palatal efficiency for blowing is found by dividing the highest "unaided" score by the highest score for "aided" blowing, multiplied by 100 .

Complete closure for blowing is indicated by a derived palatal efficiency score of 100 per cent. The validity of this test is often open to question because of variability in patient effort, nasal obstruction or other artifacts which may affect the score. To avoid this possibility the examiner should lift the tip of the patient's nose during the test so as to prevent a valve from forming at the anterior nares.

\section{Radiology}

In the event of question as to the nature or degree of palatopharyngeal deficiency, $x$-ray should be used ${ }^{3}$ to delineate palatal movements. Fluoroscopy and cineradiography are useful, and lateral soft-tissue $x$-ray films of the head may be of great value. Such studies require the use of a head positioner so that films or tracings from them may be super. imposed for comparative study. The timing and intensity of exposure must be carefully controlled if the soft tissues of the tongue and palate are to be visualized properly. Under the right conditions of exposure a contrast medium to outline the position of the tongue, palate and pharynx will not be needed. A laminograph will provide films that are somewhat more easily interpreted than standard films.

Films should be made of the patient during a variety of activities. It is necessary to expose the film during the sustained production of the activity, because otherwise it will not reveal the true dynamics of the palatopharyngeal activity. Where available, fluoroscopic examination and cinefluorographic recordings are useful. Although these provide a record of relative movements of the oral structures, the tissue definition is often inferior to shat obtained by sill x-rays.

\section{Final Comments}

The tests described in this paper employ readily available office equipment and relatively simple procedures. They take little time to administer and can contribute dynamic in- 


\section{BLOOMER AND WOLSKI}

formation concerning the reasons behind the patient's abnormal voice quality and misarticulation of speech.

Long experience of the authors in the study of palatopharyngeal activity suggests that no single testing procedure provides a reliable basis for evaluating palatopharyngeal function and palatopharyngeal valve potential. It is best to employ several procedures and compare results.

When the results of the testing procedures suggest the need for further study and defi- nition of palatopharyngeal function, referrals should be made to other specialists, such as a qualified surgeon of the palate, neurologist, dental prosthetist and speech pathologist.

\section{References}

1. Bloomer, H. H.: Observations on palatopharyngeal movements in speech and deglutition. J. Speech Hearing Dis. 18: 230, 1953.

2. Polvinec, S.: The physiology and pathology of the soft palate. J. Laryng. 66: 452, 1952.

3. Subtelny, J. D. and Koepp-Baker, H.: The signifcance of adenoid tissue in velopharyngeal func. tion. Plast. Reconstr, Surg. 17: 235, 1956.

\section{Hyperventilation in Children}

Failure to recognize that hyperventilation in infants and children may be due to causes other than primary cardiorespiratory disease can have catastrophic consequences. The two most serious yet least frequently diagnosed causes are overbreathing in salicylate poisoning and in diabetic ketoacidosis.

Hyperventilation may be the only clue to salicylate poisoning. Parents occasionally deny having given aspirin or the possibility that the child has had access to it. Use of a Phenistix paper-strip test on the urine may be helpful. Initially overbreathing is due to direct stimulation of the respiratory center by salicylate. A blood gas analysis at this time shows a respiratory alkalosis: the $\mathrm{P}_{\mathrm{CO} z}$ is low and the $\mathrm{pH}$ rises. If the diagnosis is not made at this stage a state of metabolic acidosis gradually develops, owing to increasing dehydration from the hyperventilation and the accumulation of pyruvate and lactate, with the additional burden of ketonemia. There is a tendency to acidemia, which in turn stimulates respiration, lowering the $\mathrm{P}_{\mathrm{Co}_{2}}$ further. The acid-base state of the patient cannot be assessed safely by the estimation of the plasma bicarbonate alone, as it will be reduced in both phases of the illness.

Hyperventilation in diabetic ketoacidosis of childhood is usually accompanied by marked dis curbances in hydration, and the patient may become morbund rapidly. overbreathing in this situation is the end result of acidemia and of the effort to correct this by blowing of $\mathrm{CO}_{2}$ derived from carbonic acid in the blood. Overbreathing is constant in diabetic acidosis, but the diagnosis may be obscured by the presence of abdominal rigidity or neck stiffness. Examination of the urine for sugar and ketones is essential.

There are other important causes of overventilation. In infants metabolic acidosis may develop rapidly after apparently trivial vomiting and diar. rhea. In a plump baby dehydration may be diff. cult to assess; he may averbreathe, and a common mistake then is to diagnose a respiratory infection. Valuable time may be lost in relying on antibiotics, whereas fluid replacement is the appropriate treatment. Hyperventilation may develop in encephalitis as a result of stimulation of the respiratory center. Hysterical overbreathing is rare in very young children but may occur in older ones. The overventilation occurs mainly in attacks, and often leads to tetany. Drugs used in the treatment of grand mal epilepsy may occasionally be responsible for hyperventilation.

Early diagnosis improves the prognosis immeasurably. Even if there are definite signs of respiratory disease the general practitioner should be caurious about diagnosing cardiorespiratory disease in an infant or child with hyperventilation who is dehydrated or is a known diabetic. The possibility thas overemitation is due to salicylates or other drugs must always be borne in mind.,-Brit. Mred. J., "Leading Article," May 4, 1968. 